\title{
微波促进下芳基硼酸合成芳香族硝基化合物的研究
}

\author{
间小惠李加荣张奇史大昕* \\ (北京理工大学化学与化工学院 北京 102488)
}

\begin{abstract}
摘要 微波促进下, 1,2 -二氯乙烷为溶剂、反应温度 $120{ }^{\circ} \mathrm{C}$ 、反应时间 $1 \mathrm{~min}$, 系列芳基嗍酸与五水合硝酸铋反应得到 芳香族硝基化合物. 相对于传统加热条件，微波促进反应具有：选择性高、时间短、副产物少等优点. 此外，研究了自 由基引发剂对该反应的影响，推测其可能是经过自由基转化.
\end{abstract}

关键词 芳基硼酸; 五水合硝酸铋; 芳香族硝基化合物; 微波加热; 自由基转化

\section{Synthesis of Nitroarenes under Microwave}

\author{
Yan, Xiaohui Li, Jiarong Zhang, Qi Shi, Daxin* \\ (School of Chemistry and Chemical Engineering, Beijing Institute of Technology, Beijing 102488)
}

\begin{abstract}
The reaction of arylboronic acids with bismuth(III) nitrate pentahydrate resulted in a series of nitroarenes when the reaction was carried out under microwave $\left(120^{\circ} \mathrm{C}\right)$ for $1 \mathrm{~min}$ in 1,2-dichloroethane. Compared with traditional heating condition, the method of microwave condition for the preparation of nitroarenes was simple, efficient, time-saving, and of less by-products. In addition, possible free radical mechanism was proposed.

Keywords arylboronic acids; bismuth(III) nitrate pentahydrate; nitroarenes; microwave; free radical conversion
\end{abstract}

芳香族硝基化合物是一类非常重要的有机化合物, 在医药、农药、染料、香料、炸药等领域有着广泛的应 用 ${ }^{[1 \sim 3]}$. 该类化合物的传统合成方法主要是混酸硝化 ${ }^{[4]}$, 其不足是区域选择性差, 官能团耐受性不好, 容易产生 异构体及氧化副产物等. 此外, 该反应后的大量废酸和 废水处理困难, 环境污染严重, 不符合绿色化学要求. 因此, 寻找一种条件温和、污染小且区域选择性好的合 成方法尤为重要. 近年来, 过渡金属催化的芳基卤代物 及拟卤代物 ${ }^{[5-8]}$ 、三氟甲磺酸酯 ${ }^{[6]}$ 到 $\mathrm{C}-\mathrm{NO}_{2}$ 的转化, 以 及芳烃碳-氢键的氧化硝化 ${ }^{[9 \sim 15]}$ 和芳基硼酸的硝化均取 得了一定进展.

研究发现，一些芳香金属化合物的原位硝化 ${ }^{[16]} 反$ 应区域选择性良好, 是一种很有效的硝化方法, 因此人 们对这种方法进行了大量研究, 比如芳基锂 ${ }^{[17]}$ 、杂芳基 锡烷 ${ }^{[18]}$ 的硝化. 但是, 芳基锂的硝化方法条件苛刻, 杂 芳基锡烷的硝化方法使用了有毒的有机锡和四氧化二 氮，且底物范围小. 鉴于芳香硼酸是一类非常有用的中 间体，在有机合成中有着广泛应用 ${ }^{[19]}$, 其中过渡金属催 化芳香嗍酸的官能团化是现代有机合成中构建碳一碳 键、碳-杂键的强有力工具 ${ }^{[20]}$. 因此, 人们对芳基硼酸的
原位硝化反应进行了研究, 并取得了不错的结果. 2000 年, Prakash 等 ${ }^{[21]}$ 首次报道了芳香嗍酸合成芳香族硝基 化合物的反应，他们以硝酸铵与三氟乙酸䣶作为硝化试 剂, 最终实现了芳香碀酸的区域选择性硝化. 之后, Prakash 等 ${ }^{[22]}$ 又报道了以硝酸银(铵)与三甲基一氯硅烷 作为硝化试剂, 实现了芳香嗍酸的原位硝化. Fu 等 ${ }^{[23]}$ 报 道了以亚硝酸钠为硝化试剂, 利用铜催化反应, 实现了 水相中芳香硼酸的原位硝化; Yan 等 ${ }^{[24]}$ 也发展了以亚硝 酸盐为硝化试剂, 利用氧化亚铜催化芳香硓酸的硝化反 应. $\mathrm{Wu}$ 等 ${ }^{[25]}$ 提出了以亚硝酸叔丁酯作为硝化试剂的原 位硝化反应，该方法无需昂贵的过渡金属催化剂，且操 作简单，但是该方法反应时间长，底物范围较窄，不适 用于杂芳基硼酸的反应; Yan 等 ${ }^{[26]}$ 也发展了一种新颖的、 绿色的硝化试剂一一亚硝酸特丁酯，且在无过渡金属催 化的条件下, 实现了芳香嗍酸的可控性亚硝化及硝化反 应. 2012 年, Maiti 等 ${ }^{[27]}$ 报道了以硝酸铋为硝化试剂, 在 强氧化剂条件下, 实现了芳基硼酸的硝化反应. 该方法 底物范围广, 可以实现芳香杂环硼酸的硝化反应. 同年, Yadav 等 ${ }^{[28]}$ 也报道了以硝酸铋为硝化试剂的反应, 该反 应没有使用硫代硫酸钾作氧化剂, 同样可以实现芳基硼

\footnotetext{
* Corresponding author. E-mail: shidaxin@bit.edu.cn

Received October 18, 2016; revised January 18, 2017; published online February 17, 2017.
} 
酸以及杂环芳基硼酸的原位硝化, 且这个反应有着更短 的反应时间, 底物范围宽广, 并且不需要催化剂. 2013 年, Yang 等 ${ }^{[29]}$ 报道了以硝酸铁为硝化试剂的反应. 该反 应类似于 Yadav 等报道的反应, 虽然反应时间比较长, 但是该反应同样不需要催化剂, 而且底物适用范围也 广.

众所周知，微波反应即是对极性有机物进行选择性 加热, 由于极性分子内电荷分布不均匀, 故其介电常数 较大, 能与微波产生较强的耦合作用, 其吸收微波后, 提高了分子的平均能量, 温度升高, 能够实现分子水平 上的扰动, 且物料受热均匀, 反应速度加快. 本文首次 将微波辐射用于芳基硼酸在五水合硝酸铋作用下制备 芳香硝化物的转化(Eq. 1). 实验表明: 微波辐射条件下, 反应选择性高、时间短、副产物少、后处理便捷.

\section{1 结果与讨论}

\section{1 条件优化}

以苯硼酸合成硝基苯的反应为模板, 研究了硝酸 盐、溶剂、反应温度及时间对反应的影响, 结果如表 1 所示.

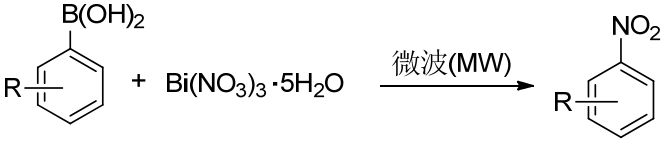

从表 1 可以看出, 在所选择的六种硝酸盐中(Entries 1 6), 五水合硝酸铋是较好的硝化试剂(Entry 1). 考虑 到微波反应对极性有机物进行选择性加热，只有具有较 大分子偶极矩的有机分子才能被微波有效加热. 由于极 性分子内电荷分布不均匀, 故其介电常数较大, 能与微 波产生较强的耦合作用, 故该反应需要在极性溶剂中进 行，从所选的四氢呋喃(THF)、乙酸乙酯、二氯甲烷、丙 酮、甲醇、乙腈与 1,2 -二氯乙烷等溶剂的实验结果 (Entries 1，7１2)不难看出，当溶剂为 1,2-二氯乙烷时, 该反应收率最高(Entry 1). 考虑到温度每升高 $10{ }^{\circ} \mathrm{C}$, 反 应速率则增加到原来的 $2 \sim 4$ 倍, 当温度由 $80{ }^{\circ} \mathrm{C}$ 升高到 $120{ }^{\circ} \mathrm{C}$ (Entries 1，15 19) 时, 反应时间相应缩短到 1 min, 且收率提高. 改变温度表明(Entries 1，13，14), $120{ }^{\circ} \mathrm{C}$ 温度下反应的收率最高为 $75 \%$ (Entry 1). 改变反 应时间可以看出(Entries 1, 20,21), 时间为 $1 \mathrm{~min}$ 时收率 最高为 $75 \%$ (Entry 1). 综上可知, 苯硼酸硝化的最优条 件为 1 equiv.的底物, 2 equiv.的五水合硝酸铋作硝化试

表 1 反应条件优化 ${ }^{a}$

Table 1 Optimization of reaction conditions

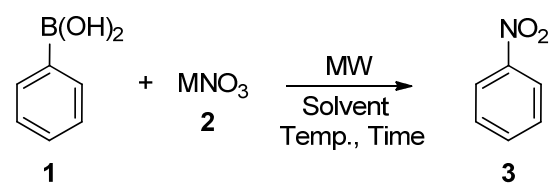

\begin{tabular}{|c|c|c|c|c|c|}
\hline Entry & Nitrate salts (equiv.) & Solvent & Temp. $/{ }^{\circ} \mathrm{C}$ & Time/min & Yield $^{b} / \%$ \\
\hline 1 & $\mathrm{Bi}\left(\mathrm{NO}_{3}\right)_{3} \cdot 5 \mathrm{H}_{2} \mathrm{O}(2)$ & $\mathrm{ClCH}_{2} \mathrm{CH}_{2} \mathrm{Cl}$ & 120 & 1 & 75 \\
\hline 2 & $\mathrm{Zn}\left(\mathrm{NO}_{3}\right)_{2} \cdot 6 \mathrm{H}_{2} \mathrm{O}(3)$ & $\mathrm{ClCH}_{2} \mathrm{CH}_{2} \mathrm{Cl}$ & 120 & 1 & Trace \\
\hline 3 & $\mathrm{KNO}_{3}(6)$ & $\mathrm{ClCH}_{2} \mathrm{CH}_{2} \mathrm{Cl}$ & 120 & 1 & 0 \\
\hline 4 & $\mathrm{NH}_{4} \mathrm{NO}_{3}(6)$ & $\mathrm{ClCH}_{2} \mathrm{CH}_{2} \mathrm{Cl}$ & 120 & 1 & 0 \\
\hline 5 & $\mathrm{AgNO}_{3}(6)$ & $\mathrm{ClCH}_{2} \mathrm{CH}_{2} \mathrm{Cl}$ & 120 & 1 & 0 \\
\hline 6 & $\mathrm{Cu}\left(\mathrm{NO}_{3}\right)_{2} \cdot 3 \mathrm{H}_{2} \mathrm{O}(3)$ & $\mathrm{ClCH}_{2} \mathrm{CH}_{2} \mathrm{Cl}$ & 120 & 1 & Trace \\
\hline 7 & $\mathrm{Bi}\left(\mathrm{NO}_{3}\right)_{3} \cdot 5 \mathrm{H}_{2} \mathrm{O}(2)$ & THF & 120 & 1 & 0 \\
\hline 8 & $\mathrm{Bi}\left(\mathrm{NO}_{3}\right)_{3} \cdot 5 \mathrm{H}_{2} \mathrm{O}(2)$ & Ethyl acetate & 120 & 1 & 0 \\
\hline 9 & $\mathrm{Bi}\left(\mathrm{NO}_{3}\right)_{3} \cdot 5 \mathrm{H}_{2} \mathrm{O}(2)$ & $\mathrm{CH}_{2} \mathrm{Cl}_{2}$ & 120 & 1 & 67 \\
\hline 10 & $\mathrm{Bi}\left(\mathrm{NO}_{3}\right)_{3} \cdot 5 \mathrm{H}_{2} \mathrm{O}(2)$ & Acetone & 120 & 1 & 0 \\
\hline 11 & $\mathrm{Bi}\left(\mathrm{NO}_{3}\right)_{3} \cdot 5 \mathrm{H}_{2} \mathrm{O}(2)$ & $\mathrm{CH}_{3} \mathrm{OH}$ & 120 & 1 & 0 \\
\hline 12 & $\mathrm{Bi}\left(\mathrm{NO}_{3}\right)_{3} \cdot 5 \mathrm{H}_{2} \mathrm{O}(2)$ & $\mathrm{CH}_{3} \mathrm{CN}$ & 120 & 1 & 0 \\
\hline 13 & $\mathrm{Bi}\left(\mathrm{NO}_{3}\right)_{3} \cdot 5 \mathrm{H}_{2} \mathrm{O}(2)$ & $\mathrm{ClCH}_{2} \mathrm{CH}_{2} \mathrm{Cl}$ & 110 & 1 & 50 \\
\hline 14 & $\mathrm{Bi}\left(\mathrm{NO}_{3}\right)_{3} \cdot 5 \mathrm{H}_{2} \mathrm{O}(2)$ & $\mathrm{ClCH}_{2} \mathrm{CH}_{2} \mathrm{Cl}$ & 130 & 1 & 67 \\
\hline 15 & $\mathrm{Bi}\left(\mathrm{NO}_{3}\right)_{3} \cdot 5 \mathrm{H}_{2} \mathrm{O}(2)$ & $\mathrm{ClCH}_{2} \mathrm{CH}_{2} \mathrm{Cl}$ & 70 & 15 & 33 \\
\hline 16 & $\mathrm{Bi}\left(\mathrm{NO}_{3}\right)_{3} \cdot 5 \mathrm{H}_{2} \mathrm{O}(2)$ & $\mathrm{ClCH}_{2} \mathrm{CH}_{2} \mathrm{Cl}$ & 80 & 15 & 58 \\
\hline 17 & $\mathrm{Bi}\left(\mathrm{NO}_{3}\right)_{3} \cdot 5 \mathrm{H}_{2} \mathrm{O}(2)$ & $\mathrm{ClCH}_{2} \mathrm{CH}_{2} \mathrm{Cl}$ & 90 & 8 & 58 \\
\hline 18 & $\mathrm{Bi}\left(\mathrm{NO}_{3}\right)_{3} \cdot 5 \mathrm{H}_{2} \mathrm{O}(2)$ & $\mathrm{ClCH}_{2} \mathrm{CH}_{2} \mathrm{Cl}$ & 100 & 4 & 75 \\
\hline 19 & $\mathrm{Bi}\left(\mathrm{NO}_{3}\right)_{3} \cdot 5 \mathrm{H}_{2} \mathrm{O}(2)$ & $\mathrm{ClCH}_{2} \mathrm{CH}_{2} \mathrm{Cl}$ & 110 & 2 & 67 \\
\hline 20 & $\mathrm{Bi}\left(\mathrm{NO}_{3}\right)_{3} \cdot 5 \mathrm{H}_{2} \mathrm{O}(2)$ & $\mathrm{ClCH}_{2} \mathrm{CH}_{2} \mathrm{Cl}$ & 120 & 2 & 63 \\
\hline 21 & $\mathrm{Bi}\left(\mathrm{NO}_{3}\right)_{3} \cdot 5 \mathrm{H}_{2} \mathrm{O}(2)$ & $\mathrm{ClCH}_{2} \mathrm{CH}_{2} \mathrm{Cl}$ & 120 & 0.5 & 42 \\
\hline
\end{tabular}

${ }^{a}$ Reaction conditions: 1 (1 mmol), 2 (2 mmol), solvent $(3 \mathrm{~mL}) .{ }^{b}$ Isolated yield. 
剂, 1,2-二氯乙烷作溶剂, 在 $120{ }^{\circ} \mathrm{C}$ 下反应 $1 \mathrm{~min}$.

为了扩大反应的适应范围, 在上述优化反应条件 下，进行了系列芳基硼酸与五水合硝酸铋的反应，结果 如表 2 所示.

由表 2 可以看出, 微波辐射条件下, 系列芳香硼酸 与五水合硝酸铋的反应状况良好，基本在 $50 \%$ 及以上. 此外, 该反应也受电子效应影响, 当反应物的邻对位有 供电子基时，产物的产率较高，如硝基苯(3a)产率 75\%, 4-硝基苯甲醚(3c)收率 73\%, 2-硝基甲苯(3e)收率 79\%, 2硝基菜(3f)收率 65\%等; 当反应物的对位有吸电子基时, 产物的收率较低, 如 4-硝基苯乙酮(3b)收率仅 19\%. 这 可能是因为自由基是缺电子体系, 邻位有吸电基时, 由 于吸电作用, 自由基的缺电性增加, 稳定性降低, 不利 于后续的转化; 而邻位有供电基时, 供电效应使自由基 的缺电特性得到减弱, 稳定性提高, 有利于后续的转化.
之后，对传统加热与微波辐射条件下的反应进行了 比较, 结果如表 3 所示. 由该表可以看出, 加热条件下 反应时间为 $1 \mathrm{~h}$ 时，反应收率很明显低于微波条件下的 反应收率，且微波反应时间更短，副产物少，分离提纯 容易.

\section{2 自由基引发剂对该反应的影响}

本文也对反应进行初步的探索，即以苯硼酸合成硝 基苯为例，进行了自由基引发剂对该反应影响的研究， 结果如表 4 所示.

我们知道, 自由基引发剂包括高温引发剂和中温引 发剂, 其中, 叔丁基过氧化氢(5)属于高温 $\left(>100{ }^{\circ} \mathrm{C}\right)$ 引 发剂, $\mathrm{K}_{2} \mathrm{~S}_{2} \mathrm{O}_{8}$ (6)属于中温 $\left(40 \sim 100{ }^{\circ} \mathrm{C}\right)$ 引发剂. 由表 4 可以看出, 高温 $\left(120{ }^{\circ} \mathrm{C}\right)$ 条件下, 添加叔丁基过氧化氢 (5)后，与添加自由基抑制剂 TEMPO (4)的反应相比，硝

表 2 芳香硝化物的合成 ${ }^{a}$

Table 2 Synthesis of nitroarenes

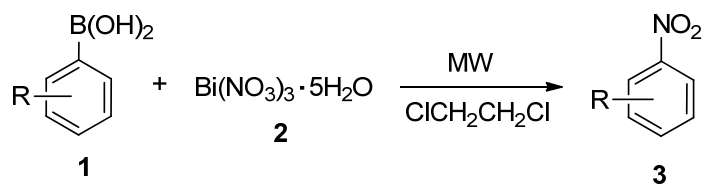

\begin{tabular}{|c|c|c|c|c|c|}
\hline Entry & Product & Yield $^{b} / \%$ & Entry & Product & Yield $^{b} / \%$ \\
\hline 1 & $3 a$ & 75 & $8^{e}$ & $3 h$ & 60 \\
\hline 2 & & 19 & 9 & $3 \mathbf{i}$ & Trace \\
\hline 3 & $3 c$ & $73^{c}$ & 10 & & Trace \\
\hline 4 & $3 d$ & 29 & 11 & & 0 \\
\hline 5 & & $79^{d}$ & 12 & & 0 \\
\hline 6 & $3 f$ & 65 & 13 & & 0 \\
\hline $7^{c}$ & $\mathbf{3 g}$ & 50 & & & \\
\hline
\end{tabular}

${ }^{a}$ Reaction conditions: $1(1 \mathrm{mmol}), 2(2 \mathrm{mmol}), 1,2$-dichloroethane $(3 \mathrm{~mL}), 120{ }^{\circ} \mathrm{C}, 1 \mathrm{~min} .{ }^{b}$ Isolated yield. ${ }^{c} 70{ }^{\circ} \mathrm{C}, 15$ min. ${ }^{d} 120{ }^{\circ} \mathrm{C}, 1 \mathrm{~min}$ later, and then $130{ }^{\circ} \mathrm{C}, 1$ $\min .{ }^{e} 130{ }^{\circ} \mathrm{C}, 1 \mathrm{~min}$ later, and then $140{ }^{\circ} \mathrm{C}, 1 \mathrm{~min}$. 
表 3 传统加热与微波条件下的反应比较 ${ }^{a}$

Table 3 Comparison of reaction carried out under traditional heating and microwave condition

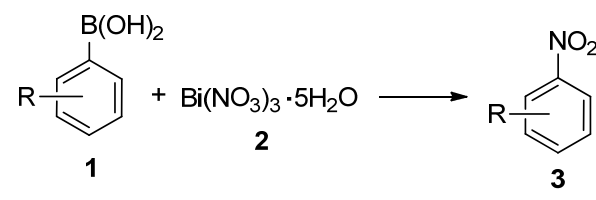

\begin{tabular}{|c|c|c|c|c|}
\hline \multirow{3}{*}{ Entry } & \multirow{3}{*}{ Product } & \multicolumn{3}{|c|}{ Yield $^{b} / \%$} \\
\hline & & \multicolumn{2}{|c|}{ Traditional heating } & \multirow{2}{*}{ MW condition } \\
\hline & & $2 \mathrm{~h}$ & $1 \mathrm{~h}$ & \\
\hline 1 & & $44(5 \mathrm{~h})$ & 10 & 75 \\
\hline 2 & $3 c$ & $60(5 \mathrm{~h})$ & 20 & $73^{c}$ \\
\hline 3 & & 67 & 35 & $79^{d}$ \\
\hline 4 & & 70 & 37 & 65 \\
\hline 5 & & 50 & 28 & $50^{c}$ \\
\hline
\end{tabular}

${ }^{a}$ Heating reaction conditions: $\mathbf{1}(1 \mathrm{mmol}), \mathbf{2}(2 \mathrm{mmol})$, toluene $(5 \mathrm{~mL}), 80{ }^{\circ} \mathrm{C}$, $2 \mathrm{~h}$ or $1 \mathrm{~h}$, Ar. Microwave reaction conditions: $1(1 \mathrm{mmol}), 2(2 \mathrm{mmol})$, 1,2-dichloroethane $(3 \mathrm{~mL}), 120{ }^{\circ} \mathrm{C}, 1 \mathrm{~min} .{ }^{b}$ Isolated yield. ${ }^{c} 70{ }^{\circ} \mathrm{C}, 15 \mathrm{~min}$. ${ }^{d} 120{ }^{\circ} \mathrm{C}, 1 \mathrm{~min}$ later, and then $130{ }^{\circ} \mathrm{C}, 1 \mathrm{~min}$.

表 4 自由基引发剂对该反应的影响 $1^{a}$

Table 4 the Influence of free radical initiator 1

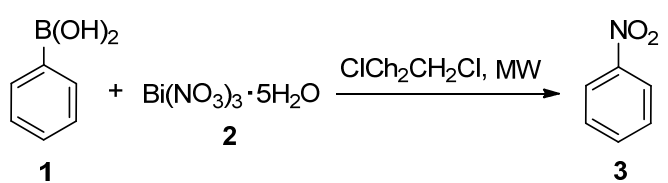

\begin{tabular}{cccc}
\hline Entry & Conditions & Temp. $/{ }^{\circ} \mathrm{C}$ & Yield $^{b} / \%$ \\
\hline 1 & TEMPO (4) & 120 & 10 \\
2 & tert-butyl hydroperoxide (5) & 120 & 83 \\
3 & $\mathrm{~K}_{2} \mathrm{~S}_{2} \mathrm{O}_{8}(\mathbf{6})$ & 80 & 10 \\
\hline
\end{tabular}

${ }^{a}$ Reaction conditions: 1 (1 mmol), 2 (2 mmol), 4 (3 mmol), 5 (65\% aq. 2 droups), 6 (1 mmol), solvent ( $3 \mathrm{~mL}), 1$ h. ${ }^{b}$ Isolated yield.

基苯的收率由 $10 \%$ 上升到了 $83 \%$, 这可能是因为自由基 捕捉剂 TEMPO 的加入 ${ }^{[27]}$, 抑制了反应过程中自由基的 产生, 因此反应难以进行, 最终收率过低, 而叔丁基过 氧化氢的加入, 促进了自由基的产生, 故而收率升高. 中温 $\left(80{ }^{\circ} \mathrm{C}\right)$ 条件下, 添加 $\mathrm{K}_{2} \mathrm{~S}_{2} \mathrm{O}_{8}(6)$ 后, 与添加自由基 抑制剂 TEMPO (4)的反应相比, 硝基苯的收率基本没有 变化, 这可能是因为中温引发剂 $\mathrm{K}_{2} \mathrm{~S}_{2} \mathrm{O}_{8}(\mathbf{6})$ 在该条件下,
促进自由基产生需要一定的时间，故短时间内反应难以 进行, 收率较低.

此外，我们也对不同温度下的自由基引发剂对反应 的促进效果进行了探究，以添加叔丁基过氧化氢为例， 结果见表 5.

表 5 自由基引发剂对反应的影响 $2^{a}$

Table 5 the Influence of free radical initiator 2

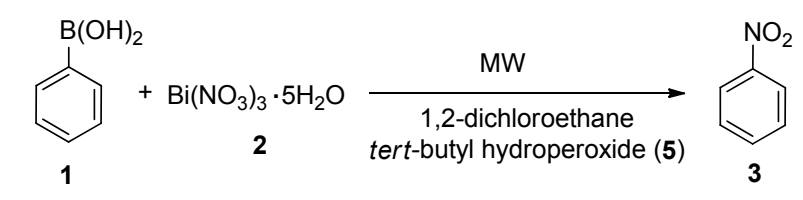

\begin{tabular}{ccc}
\hline Entry & Temp. $/{ }^{\circ} \mathrm{C}$ & Yield $^{b} / \%$ \\
\hline 1 & 140 & 92 \\
2 & 120 & 83 \\
3 & 80 & 0 \\
\hline
\end{tabular}

${ }^{a}$ Reaction conditions : 1 (1 mmol), 2 (2 mmol), 5 (65\% aq. 2 drops), solvent (3 $\mathrm{mL}), 1 \mathrm{~h} .{ }^{b}$ Isolated yield.

从表 5 中可以看出, 与 $120{ }^{\circ} \mathrm{C}$ 条件下的反应相比, 温度升高到 $140{ }^{\circ} \mathrm{C}$ 时, 硝基苯的收率由 $83 \%$ 升高到了 $92 \%$, 这可能是因为高温引发剂在温度较高时, 分解速 率快，促进了反应过程中自由基的产生，进而加快了反 应速率，使收率提高; 温度过低 $\left(80{ }^{\circ} \mathrm{C}\right)$ 时，硝基苯的收 率由 $83 \%$ 下降到了 0 , 这可能是因为反应温度过低时, 自由基引发剂分解速率过低, 导致反应所需时间变长, 因此反应收率降低.

综上所述，自由基引发剂对该反应有很明显的影 响, 推测其机理可能是经过自由基转化.

\section{2 结论}

本实验在微波辐射条件下进行了芳基硼酸与五水 合硝酸铋的反应，得到了系列芳香族硝基化合物，通过 对不同反应条件的探讨，确定了最佳反应条件. 当微波 反应硝化试剂为五水合硝酸铋、溶剂为 1,2 -二氯乙烷、 温度为 $120{ }^{\circ} \mathrm{C}$ 、反应时间为 $1 \mathrm{~min}$ 时, 收率最高可达 $79 \%$. 同时, 与传统加热条件下的反应结果相比, 微波 反应选择性高、时间短、副产物少、易分离提纯. 最后, 对自由基引发剂对该反应的影响进行了探索，推测其可 能是经过自由基转化.

\section{3 实验部分}

\section{1 仪器与试剂}

ZF-1 型三用紫外仪, ETS-D5 型恒温磁力加热搅拌 器, XT4 型熔点测定仪, YP2002 型电子天平, SHB-III型 循环水式真空原, RE-52AA 型旋转蒸发器, DZF-6030A 真空干燥箱, Boitage 型微波合成反应仪, $400 \mathrm{MHz}$ 核磁 
共振仪(Bruker ARX 400), Perkin Elmer FT-IR Spectrophotometer 型红外仪, 所用试剂均为分析纯.

\section{2 实验方法}

在放有磁子的微波管中加入化合物 $1(1 \mathrm{mmol})$ 和五

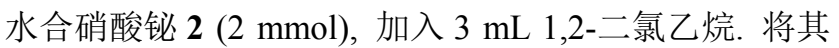
加硅胶垫及铝盖密封后, 放入微波反应器中, 设置温度 为 $120{ }^{\circ} \mathrm{C}$, 时间为 $1 \mathrm{~min}$. 反应结束后, 待微波管冷却至 室温, 将混合物减压抽滤, 滤饼用乙酸乙酯和二氯甲烷 洗涤, 收集滤液, 减压旋蒸, 进行柱层析分离得到目标 产物 3, 洗脱剂为 $[V$ (乙酸乙酯) : $V$ (石油醚 $)=5: 95]$. 并用 ${ }^{1} \mathrm{H}$ NMR、 ${ }^{13} \mathrm{C} N M R$ 和红外对目标化合物进行了表 征.

硝基苯 (3a) $)^{[27]}$ : 淡黄色油状液体. ${ }^{1} \mathrm{H}$ NMR (400 $\left.\mathrm{MHz}, \mathrm{CDCl}_{3}\right) \delta: 8.22(\mathrm{~d}, J=8.0 \mathrm{~Hz}, 2 \mathrm{H}), 7.71$ (t, $J=8.0$ $\mathrm{Hz}, 1 \mathrm{H}), 7.55$ (t, $J=8.0 \mathrm{~Hz}, 2 \mathrm{H}) ;{ }^{13} \mathrm{C}$ NMR $(100 \mathrm{MHz}$, $\left.\mathrm{CDCl}_{3}\right) \delta: 148.24,134.65,129.36,123.48$. IR (KBr) $v$ : $3071,1605,1524,1348 \mathrm{~cm}^{-1}$.

4-硝基苯乙酮 $(3 \mathbf{b}){ }^{[28]}$ : 黄色固体, m.p. $74 \sim 76{ }^{\circ} \mathrm{C}$ (Lit. ${ }^{[28]} 75 \sim 76{ }^{\circ} \mathrm{C}$ ); ${ }^{1} \mathrm{H}$ NMR (400 MHz, DMSO) $\delta: 8.34$ (d, $J=8.0 \mathrm{~Hz}, 2 \mathrm{H}), 8.18$ (d, $J=8.0 \mathrm{~Hz}, 2 \mathrm{H}), 2.68$ (s, 3H); ${ }^{13} \mathrm{C}$ NMR (101 MHz, DMSO) $\delta: 197.14,149.64,141.01$, 129.36, 123.60, 26.89; IR (KBr) v: 3600, 3107, 1693, $1606,1528,1345,1261 \mathrm{~cm}^{-1}$.

4-硝基苯甲醚(3c) ${ }^{[28]}$ : 黄白色固体, m.p. $52 \sim 55{ }^{\circ} \mathrm{C}$ (Lit. ${ }^{[28]} 51 \sim 53{ }^{\circ} \mathrm{C}$ ); ${ }^{1} \mathrm{H}$ NMR (400 MHz, $\mathrm{CDCl}_{3}$ ) $\delta: 8.19$ (d, $J=8.0 \mathrm{~Hz}, 2 \mathrm{H}), 6.96$ (d, $J=8.0 \mathrm{~Hz}, 2 \mathrm{H}), 3.89$ (s, 3H); ${ }^{13} \mathrm{C}$ NMR $\left(100 \mathrm{MHz}, \mathrm{CDCl}_{3}\right) \delta 164.68,141.65,125.98$, 114.10, 56.04; IR (KBr) v: 3433, 3081, 1608, 1591, 1499, $1332,1264,1110,1022 \mathrm{~cm}^{-1}$.

4-硝基甲苯 (3d) ${ }^{[28]}$ : 淡黄色固体, m.p. $51 \sim 53{ }^{\circ} \mathrm{C}$ (Lit. $\left.{ }^{[28]} 51 \sim 52{ }^{\circ} \mathrm{C}\right),{ }^{1} \mathrm{H}$ NMR $\left(400 \mathrm{MHz}, \mathrm{CDCl}_{3}\right) \delta: 8.11$ (d, $J=8.0 \mathrm{~Hz}, 2 \mathrm{H}), 7.31$ (d, $J=8.0 \mathrm{~Hz}, 2 \mathrm{H}), 2.46$ (s, 3H); ${ }^{13} \mathrm{C}$ NMR $\left(100 \mathrm{MHz}, \mathrm{CDCl}_{3}\right) \delta: 145.98,145.78,129.46$, 123.23, 21.56; IR (KBr) v: 3649, 3081, 1597, 1517, 1345 $\mathrm{cm}^{-1}$.

2-硝基甲苯 (3e $)^{[28]}$ : 淡黄色液体. ${ }^{1} \mathrm{H}$ NMR (400 MHz, DMSO- $\left.d_{6}\right) \delta: 7.98(\mathrm{~d}, J=8.0 \mathrm{~Hz}, 1 \mathrm{H}), 7.64(\mathrm{t}, J=$ $8.0 \mathrm{~Hz}, 1 \mathrm{H}), 7.52 \sim 7.45(\mathrm{~m}, 2 \mathrm{H}), 2.52(\mathrm{~s}, 3 \mathrm{H}) ;{ }^{13} \mathrm{C} \mathrm{NMR}$ (101 MHz, DMSO) $\delta$ : 154.25, 138.69, 138.06, 137.93, $132.63,129.60,24.75$; IR (KBr) v: 3465, 3069, 1612, $1523,1347 \mathrm{~cm}^{-1}$.

2-硝基萫 (3f ${ }^{[6]}$ : 淡黄色固体, m.p. $76 \sim 78{ }^{\circ} \mathrm{C}$ (Lit. ${ }^{[6]} 74 \sim 76{ }^{\circ} \mathrm{C}$ ); ${ }^{1} \mathrm{H}$ NMR (400 MHz, DMSO) $\delta: 9.01$ (s, 1H), $8.31-8.12(\mathrm{~m}, 4 \mathrm{H}), 7.82-7.72(\mathrm{~m}, 2 \mathrm{H}) ;{ }^{13} \mathrm{C}$ NMR $\left(101 \mathrm{MHz}\right.$, DMSO- $\left.d_{6}\right) \delta 145.15,135.56,131.70$,
130.28, 130.15, 129.94, 128.17, 128.06, 124.70, 119.13; IR (KBr) $v: 3674,3062,1603,1529,1500,1347,1333 \mathrm{~cm}^{-1}$.

3，4-亚甲二氧基硝基苯 $(\mathbf{3 g})^{[28]}$ : 淡黄色固体, m.p. $144 \sim 147{ }^{\circ} \mathrm{C}$ (Lit. ${ }^{[28]} 146 \sim 147{ }^{\circ} \mathrm{C}$ ); ${ }^{1} \mathrm{H}$ NMR $(400 \mathrm{MHz}$, $\left.\mathrm{CDCl}_{3}\right) \delta: 7.89(\mathrm{~d}, J=8.0 \mathrm{~Hz}, 1 \mathrm{H}), 7.67(\mathrm{~s}, 1 \mathrm{H}), 6.87(\mathrm{~d}$, $J=8.0 \mathrm{~Hz}, 1 \mathrm{H}), 6.14(\mathrm{~s}, 2 \mathrm{H}) ;{ }^{13} \mathrm{C} \mathrm{NMR}\left(100 \mathrm{MHz}, \mathrm{CDCl}_{3}\right)$ $\delta: 153.15,148.25,142.97,119.91,107.58,104.53,103.05$; IR (KBr) v: 3435, 3116, 2924, 1609, 1503, 1487, 1339 $\mathrm{cm}^{-1}$.

4-硝基二苯并噻吩 $(\mathbf{3 h})^{[27]}$ : 黄色固体, m.p. 159 $161{ }^{\circ} \mathrm{C}$ (Lit. ${ }^{[27]} 158 \sim 161{ }^{\circ} \mathrm{C}$ ); ${ }^{1} \mathrm{H}$ NMR $(400 \mathrm{MHz}$, $\left.\mathrm{CDCl}_{3}\right) \delta: 8.46 \sim 8.40(\mathrm{~m}, 2 \mathrm{H}), 8.16(\mathrm{~d}, J=8.0 \mathrm{~Hz}, 1 \mathrm{H})$, $7.91(\mathrm{~d}, J=8.0 \mathrm{~Hz}, 1 \mathrm{H}), 7.61 \sim 7.50(\mathrm{~m}, 3 \mathrm{H}) ;{ }^{13} \mathrm{C} \mathrm{NMR}$ $\left(100 \mathrm{MHz}, \mathrm{CDCl}_{3}\right) \delta: 142.89,140.94,138.86,135.41$, $133.81,128.15,127.21,125.28,124.67,123.22,122.73$, 121.83; IR (KBr) v: 3434, 2921, 1610, 1520, 1340, 1318 $\mathrm{cm}^{-1}$.

辅助材料(Supporting Information) 硝基苯、4-硝基苯 乙酮、4-硝基苯甲醚、4-硝基甲苯、2-硝基甲苯、2-硝 基萗、3,4-亚甲二氧基硝基苯、4-硝基二苯并噻吩的 IR, ${ }^{1} \mathrm{H}$ NMR, ${ }^{13} \mathrm{C}$ NMR 图. 这些材料可以免费从本刊网站 (http://sioc-journal.cn/)上下载.

\section{Referenes}

[1] Muller, W. A. The Benzodiazepine Receptor, Cambridge University Press, New York, 1988.

[2] Zollinger, H. Color Chemistry, Wiley-VCH, New York, 1987, 161.

[3] Belciug, M.; Ananthanarayanan, V. S. J. Med. Chem. 1994, 37, 4392.

[4] Ono, N. The Nitro Group in Organic Synthesis, Wiley-VCH, New York, 2001.

[5] Saito, S.; Koizumi, Y. Tetrahedron Lett. 2005, 46, 4715.

[6] Fors, B. P.; Buchwald, S. L. J. Am. Chem. Soc. 2009, 131, 12898.

[7] LaBeaume, P.; Placzek, M.; Daniels, M.; Kendrick, I.; Ng, P.; McNeel, M.; Afroze, R.; Alexander, A.; Thomas, R.; Kallmerten, A. E.; Jones, G. B. Tetrahedron Lett. 2010, 51, 1906.

[8] Joseph, P. J. A.; Priyadarshini, S.; Kantam, M. L.; Maheswaran, H. Tetrahedron Lett. 2012, 53, 1511.

[9] Liu, Y.; Lou, S.; Xu, D.; Xu, Z. Chem. Eur. J. 2010, 16, 13590.

[10] Zhang, L.; Liu, Z.; Li, H.; Fang, G.; Barry, B. D.; Belay, T. A.; Bi, X.; Liu, Q. Org. Lett. 2011, 13, 6536.

[11] Xie, F.; Qi, Z. S.; Li, X. W. Angew. Chem., Int. Ed. 2013, 52, 11862.

[12] Zhang, W.; Lou, S. J.; Liu, Y. K.; Xu, Z. Y. J. Org. Chem. 2013, 78, 5932.

[13] Zhang, W.; Zhang, J.; Ren, S. B.; Liu, Y. K. J. Org. Chem. 2014, 79 11508 .

[14] Katayev, D.; Pfister, K. F.; Wendling, T.; Gooßen, L. J. Chem. Eur J. 2014, 20, 9902.

[15] Majhi, B.; Kundu, D.; Sabir, A.; Brindaban, C. R. Chem. Eur. J. 2014, 20, 9862.

[16] Yan, G. B.; Yang, M. H. Org. Biomol. Chem. 2013, 11, 2554.

[17] Tani, K.; Lukin, K.; Eaton, P. E. J. Am. Chem. Soc. 1997, 119, 1476.

[18] Fargeas, V.; Favresse, F.; Mathieu, D.; Beaudet, I.; Charrue, P.; Lebret, B.; Piteau, M.; Quintard, J. Eur. J. Org. Chem. 2003, 1711.

[19] Hall, D. G. Boronic Acids: Preparation, Applications in Organic 
Synthesis and Medicine, Wiley-VCH, Weinheim, 2005.

[20] Miyaura, N.; Suzuki, A. Chem. Rev. 1995, 95, 2457.

[21] Salzbrunn, S.; Simon, J.; Prakash, G. K. S.; Petasis, N. A.; Olah, G. A. Synlett 2000, 1485.

[22] Prakash, G. K. S.; Panja, C.; Mathew, T.; Surampudi, V.; Petasis, N. A.; Olah, G. A. Org. Lett. 2004, 6, 2205.

[23] Yang, H. J.; Li, Y.; Jiang, M.; Wang, J. M.; Fu, H. Chem. Eur. J. 2011, 17, 5652 .

[24] Yan, G.; Zhang, L.; Yu, J. Lett. Org. Chem. 2012, 9, 133.

[25] Wu, X.; Schranck, J.; Neumann, H.; Beller, M. Chem. Commun.
2011, 47, 12462

[26] Wang, S.; Shu, C.; Wang, T.; Yu, J.; Yan, G. Chin. Chem. Lett. 2012, 23,643 .

[27] Manna, S.; Maity, S.; Rana, S.; Agasti, S.; Maiti, D. Org. Lett. 2012, 14, 1376.

[28] Yadav, R. R.; Vishwakarma, R. A.; Bharate, S. B. Tetrahedron Lett. 2012, 53, 5958.

[29] Jiang, M.; Yang, H. J.; Li, Y.; Jia, Z. Y.; Fu. H. RSC Adv. 2013, 3, 25902

(Cheng, F.) 